\title{
A PROPOSAL TO USE THE TAGUI FRAMEWORK AS AN RPA TOOL
}

\section{Mauro Henrique da Silva Wencelewski ${ }^{1}$, Priscila Reis Soares da Paixão ${ }^{2}$, Manoel Henrique Reis Nascimento $^{3}$}

\footnotetext{
1,2,3 Fundação Centro de Análise Pesquisa e Inovação Tecnológica (FUCAPI) - Av. Gov. Danilo de Matos Areosa, 381 - Distrito Industrial, Manaus - AM.
}

Email: mauro.wencelewski@gmail.com, priscila.wencelewski@gmail.com, hreys@ bol.com

Received: June 03th, 2019

Accepted: June 06 ${ }^{\text {th }}, 2019$

Published: September $30^{\text {th }}$, 2019

Copyright @2016 by authors and Institute of Technology Galileo of Amazon (ITEGAM). This work is licensed under the Creative Commons Attribution International License (CC BY 4.0). https://creativecommons.org/lice nses/by/4.0/

\begin{abstract}
The automation of processes is an increasingly common reality today, the "digital" employee is increasingly present in companies of various segments. The purpose of this paper is to contextualize the Robotic Process Automation technology as well as to present an open-source framework called TagUI as a tool option. In a way that enables and democratizes the use of technology in other market segments or even by private users. As a prototype and case study we performed automation of a process that fits in the feasibility prerequisites for automation. Finally, we draw a parallel between the benefits expected to be achieved with the use of RPA and those achieved after the deployment of the developed robot.
\end{abstract}

Keywords: Robotic Process Automation, RPA, Process Automation.

\section{UMA PROPOSTA DE USO DO FRAMEWORK TAGUI COMO FERRAMENTA DE RPA}

\begin{abstract}
RESUMO
A automação de processos é uma realidade cada vez mais comum atualmente, o funcionário "digital" está cada vez mais presente em empresas de diversos segmentos. A proposta desse trabalho é contextualizar a tecnologia de Robotic Process Automation (RPA), assim como apresentar um framework open-source chamado TagUI como uma opção de ferramenta. De forma viabilizando e democratizando o uso da tecnologia em outros segmentos de mercado ou ainda por usuários particulares. Como protótipo e estudo de caso foi realizada automação de um processo que se encaixa nos pré-requisitos de viabilidade para automação. Por fim, traçando um paralelo entre os benefícios que esperam ser alcançados com o uso de RPA e os alcançados após a implantação do robô desenvolvido.
\end{abstract}

Keywords: Robotic Process Automation, TagUI, Automação de Processos.

\section{INTRODUÇÃO}

De acordo com um estudo conduzido pela [1], a maior parte das grandes corporações existentes hoje, usam milhares de planilhas e bancos de dados Access para monitorar e coordenar seus trabalhos.

E alguns Setores de Tecnologia da Informação (TI) dedicam algo entre $30 \%-70 \%$ dos seus esforços e orçamentos para manter seus sistemas legados [2].
O motivo geralmente é a falta de métodos de integração para conectar sistemas existentes forçam os usuários de negócio a criarem suas próprias soluções alternativas.

Similarmente a robotização das linhas de produção na década de 90, corporações estão iniciando a jornada de transformação robótica/revolução digital [3]. Essa revolução está tomando grandes proporções porque os usuários/clientes querem operar digitalmente. O motivo é que eles gostariam de utilizar tecnologias iterativas, aplicativos móveis, mídias sociais, texto, conversas online e etc. As pessoas estão acostumadas a usar 
tecnologias sofisticadas nas suas vidas pessoais, e agora, esperam utilizar esse mesmo tipo de tecnologia nas suas vidas profissionais [4].

Com a evolução das tecnologias como inteligência artificial, visão computacional, chat bots e ferramentas de automação de software, foi um caminho quase que natural a busca de grandes corporações por alternativas a integração tradicional de sistemas e automação de tarefas operacionais.

Robotic Process Automation (RPA) pode ser a conexão entre sistemas legados antiquados no back office e sistemas de interação com cliente no front office, servindo como fundação para automação inteligente de processos digitais no toolkit dos futuros empregos [4].

De acordo com [5] as empresas estão começando a empregar RPA junto com tecnologias cognitivas como reconhecimento de fala, processamento de linguagem natural e aprendizado de máquina para automatizar uma parte das atividades onde eram necessários julgamentos humanos. Essa integração está levando RPA a um novo patamar na área de automação de processos, e como resultado, ajudando empresas a se tornarem mais ágeis e eficientes.

Nesse contexto, é possível afirmar que o mercado é dominado por ferramentas comerciais e de código fechado. Assim como, em todas é necessário aquisição de algum tipo de licença comercial, que em geral tem um custo de alguns milhares de dólares por robô.

Dessa forma, existe um gap no mercado, para pequenos e médios negócios. Ou ainda para uso pessoal, restando a usuários domésticos utilizarem licenças acadêmicas e as pequenas empresas procurar as próprias soluções alternativas.

A proposta desse trabalho, é demonstrar uma possível solução a esses casos com o uso de uma ferramenta open-source, em um caso de uso de automação de um processo pequeno.

\section{REFERENCIAL TEÓRICO II. 1 ROBOTIC PROCESS AUTOMATION}

Robotic Process Automation (RPA) é um software configurado para simular ações humanas com base em regras préestabelecidas. Definido por [6] como uma instância de software pré-configurada que usa regras de negócios e coreografia de atividade predefinida para concluir execução autônoma de uma combinação de processos, atividades, transações e tarefas em um ou mais sistemas não relacionados para fornecer um resultado ou serviço com exceções gerenciadas por humanos.

De acordo com [7], para processos de negócio RPA é mais comumente referenciado com um software que realiza atividades que anteriormente eram executadas por humanos. Como por exemplo, transferir dados de múltiplas interfaces de entrada, como e-mails e planilhas para sistemas ERP (Enterprise Resource Planning) e sistemas CRM (Customer Relationship Management). Outra definição encontrada é de acordo com [5], é que RPA é a combinação de inteligência artificial e automação, dessa forma permitindo aos funcionários de uma empresa configurar um software ou um "robô" para decidir, coletar e extrair conhecimento, assim como, reconhecer padrões, aprender e se adaptar a novas situações ou ambientes.

Embora o termo robô denote visões de máquinas eletromecânicas que executam tarefas humanas, o termo no que se refere à automação de serviços refere-se a algo menos ameaçador: um software que executa as tarefas repetitivas e monótonas, anteriormente realizada por seres humanos, para que os seres humanos possam se concentrar em tarefas com informações mais desestruturadas e interessantes [8].
Nós podemos usar robôs para amplificar e melhorar qualidades humanas, permitindo grandes ganhos econômicos e trabalhos mais satisfatórios [8].

RPA possui características únicas, que o diferencia de outros paradigmas de automação existentes na automação de processos de negócio, reengenharia de processos ou gerenciamento de sistemas de processos. Primeiramente, robôs RPA realizam as tarefas da mesma forma que um usuário realizam, através da camada de apresentação [6].

Ainda de acordo com [6] robôs RPA podem ser comparados macros de Excel que automatizam tarefas especificas. A principal diferença entre RPA e "macros" é que RPA pode ser gravado para ser utilizado com qualquer aplicação instalada no computador ou servidor.

Para entender melhor, a definição de RPA, é interessante contextualizar a história da evolução da tecnologia. De acordo com [9] após a década de 90 começaram a surgir desenvolvimento de tecnologias que precederam o RPA.

\section{Screen Scraping}

Essa tecnologia surgiu antes do desenvolvimento da internet, onde foi a primeira tecnologia criar uma ponte entre antigos sistemas legados e sistemas modernos, mais recentemente vem sendo utilizada para extrair informações da web na camada de apresentação [9]. Mas, no entanto, ainda de acordo com [9], mesmo com todos os benefícios trazidos por essa tecnologia sob o trabalho manual, ainda é uma tecnologia limitada, pois a compatibilidade do software com sistemas existentes e com o HTML de aplicações web traz uma dificuldade que um usuário de negócio comum não consegue compreender.

\section{Automação e Gerenciamento de Fluxo de Trabalho}

Enquanto o termo Workflow Automation remete a década de 1920, onde durante a era industrial e o surgimento da manufatura a automação de processos de software, pode por exemplo, ajudar o processamento de pedidos de compra, capturando determinados campos, como informações do cliente, total da compra e itens comprados, depois convertendo-os e inserindo-os no banco de dados da empresa e notificando o funcionário responsável [9]. É um tipo de ferramenta que elimina trabalhos manuais e repetitivos.

\section{Inteligência Artificial}

O termo "inteligência artificial" foi originalmente cunhado em 1956 numa conferência em Darthmouth College. Inteligência Artificial (IA) é a habilidade de sistemas computacionais realizarem tarefas que normalmente iriam requerer intervenção e raciocínio humano [9]. Apesar de ser uma tecnologia que ainda é cara, as vantagens que podem ser obtidas com seu uso incluem o aumento na assertividade em execução de tarefas e substituição em tarefas tediosas, demoradas e manuais.

\section{Machine Learning}

Como descrito por [10], Machine Learning (ML) se refere a habilidade de um sistema computacional de melhorar sua performance ao se expor a dados sem a necessidade de seguir regras programadas explicitamente. No seu centro, ML é o processo de descoberta automática de padrões em dados. Uma vez descoberto, o padrão pode ser usado para realizar predições. As aplicações para essa tecnologia são amplas, e com potencial de melhorar performance em qualquer atividade que lide com grandes quantidades de dados.

$\mathrm{O}$ uso de IA e Machine Learning (ML), permite que o RPA realize tarefas de maneira muito mais robusta. Por exemplo, 
o robô deve continuar funcionando quando uma interface web de alguma aplicação for modificado. $\mathrm{O}$ uso de IA/ML ajuda a identificar mudanças na interface, portanto, sendo bem diferente da tradicional screen scraping [11].

De acordo com [9] o surgimento do termo RPA vem do inicio dos anos 2000. RPA é uma tecnologia em evolução, mas que se baseia em conceitos de tecnologias citadas anteriormente, e eleva essas mesmas tecnologias a um novo patamar. Mesmo sendo dependente de tecnologias como Screen Scraping, as ferramentas de RPA permitem aos usuários criarem suas automações e seus fluxos de processos usando funcionalidades drag and drop de forma visual que as torna independente que conhecimento de linguagens de programação.

A tecnologia de RPA não é parte da infraestrutura de TI de uma companhia, mas ao invés, fica sob a mesma . Isso permite que as companhias implementem a tecnologia rapidamente e de maneira eficiente sem alterar a infraestrutura e sistemas já existentes. Outra visão sobre a tecnologia de RPA é considerar que não foi desenvolvida para ser uma aplicação de negócio, mas sim um intermediário para o operador humano atuar nas aplicações de negócios [12].

Então, pode se afirmar que RPA é um "robô" de software que executa tarefas repetitivas e baseadas em regras pré-definidas, atuando em um ou múltiplos sistemas, através da camada de apresentação, ou seja, a camada que o usuário utiliza para realizar essas tarefas manualmente.

\section{Áreas de Aplicação}

O uso de robôs está ficando cada vez mais comum no mercado atual. Ao longo dos anos, é uma tecnologia muito presente em call centers e operações bancárias de back-office. Como pode ser visto na Figura 1, o uso de RPA é mais indicado para substituir humanos em processos do tipo Swivel-Chair (Cadeira Giratória) [13]. Ou seja, processos onde o mesmo tipo de informação é inserido pelo usuário em sistemas diferentes.

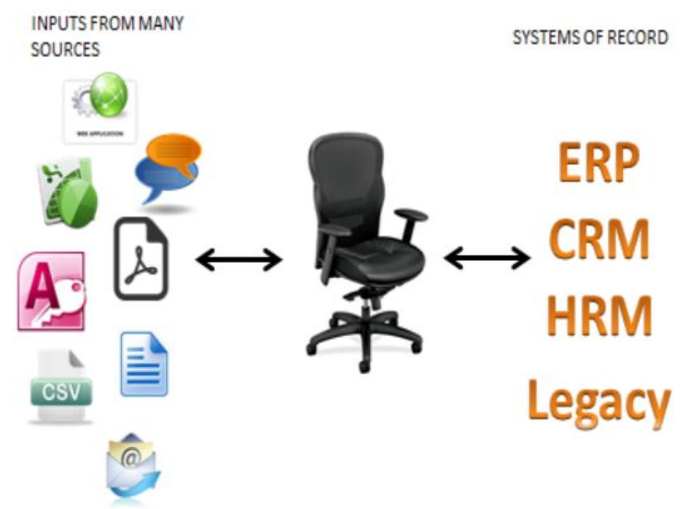

Figura 1 - Processos Swivel Chair. Fonte: [10].

Como por exemplo citado por [13], um especialista de RH responsável por fazer a admissão de novos funcionários em uma grande companhia. Esse processo geralmente pode envolver realizar logins e logoff em vários sistemas para configurar os benefícios, folha de pagamento, e-mail, caixa postal, liberações de segurança, espaço no escritório, móveis de escritório, computador, vaga de estacionamento, prestação de despesas, crachá de identificação e cartões de visita no padrão da companhia. Multiplique esse processo por milhares de funcionários admitidos por ano. Nesse caso, um RPA pode ser configurado para realizar tudo isso que um especialista de $\mathrm{RH}$ realizava, mas utilizando suas próprias credenciais no sistema.
Dessa forma, os robôs são desenvolvidos para serem consistentes com a Governança de TI e requisitos de segurança, arquitetura e infraestrutura, sendo rapidamente implantados, reutilizados e escalados [13].

Outras áreas de aplicação, de acordo com [7] são entrada de clientes, funcionários e fornecedores, geração de relatórios, processamento de folha de pagamento, monitoramento de preços e cotações, sistemas CRM, processamento de pedidos de compras, agendamento de entregas e rastreio de pedidos.

Idealmente, existem algumas características de processos que podem ser automatizados obtendo o máximo da tecnologia. São processos em geral com:

- Grandes volumes de dados estruturados (Planilhas, bases de dados e sistemas)

- Tarefas repetitivas e com regras bem definidas.

- Estáveis

- Alto Valor

- Alto Risco

De acordo com [14] processos com grandes volumes de dados são ideais pelo tempo de processamento manual dessas informações. Enquanto processos de alto valor são ideais porque criam uma melhor experiência para o cliente e tem alto retorno para companhia. E por último, processos de alto risco são processos que podem colocar a empresa em problemas por conta de atrasos ou informações inseridas de forma incorreta em algum lugar.

\section{Tipos de Automação}

Existem duas formas de se implantar uma automação RPA: a automação assistida, representada pela Figura 2, onde o robô automatiza aplicações que estão executando no computador do funcionário. Ou automação não-assistida, representada pela Figura 3, onde o robô é instalado em diversos computadores sem a intervenção humana.

\section{- Assistida}

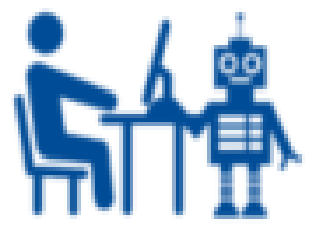

\section{Assisted Automation}

\author{
Figura 2 - Automação Assistida. \\ Fonte: [4].
}

Segundo [4] esse tipo de automação começou como uma ferramenta para melhorar a produtividade de call centers, onde agentes iriam acionar uma série de passos automatizados através de diversas aplicações instaladas em seus computadores. Esse tipo de abordagem reduziu drasticamente o tempo de processamento, resultou em economia e melhorou a experiência do consumidor. Dessa forma, processos longos e complexos foram substituídos por simples cliques, mas um ponto de atenção desse tipo de abordagem é muito suscetível a mudanças de configurações do ambiente, como por exemplo Placas de vídeos, resoluções ou configurações de vídeo diferentes.

Automação assistida atualmente, é conhecida como Robotic Desktop Automation (RDA) e é usada para auxiliar na produtividade [4]. 


\section{- Não-Assistida}

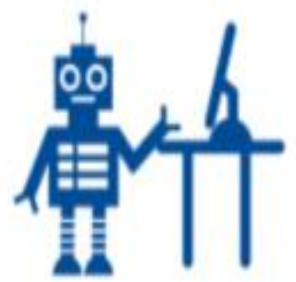

\section{Unassisted Automation}

\author{
Figura 3 - Automação Não Assistida. \\ Fonte: [4].
}

Esse tipo de automação não requer um funcionário operando o computador. O processo de início, execução e finalização pode ser automatizado e facilitado através de dashboards que fornecem uma "sala de controle" para alocar os robôs em máquinas, ajustar prioridades de execução e pilhas e intervir na performance de um robô especifico (se necessário) [4].

Mas ainda de acordo com [4] uma desvantagem significativa do uso de automação não assistida, é o fato que o robô requer dados digitalizados e estruturados, com regras bem definidas.

Em casos, onde esses pré-requisitos não podem ser atendidos dividir o processo entre execução humana e processos automatizados pode ser a melhor opção para o uso de automação não assistida [4].

Fora desse contexto, esse tipo de robô pode funcionar 24/7, somente necessitando da intervenção humana se encontrar alguma exceção não tratada.

\section{Benefícios}

Ao implantar um sistema RPA existem inúmeros benefícios que podem ser alcançados como descritos na Figura 4:

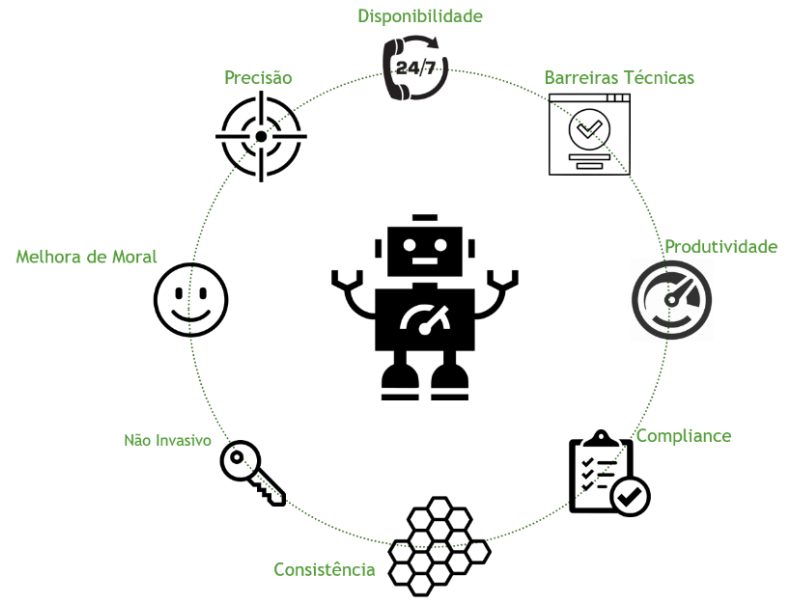

Figura 4 - Benefícios de RPA.

Fonte: Autores, (2019).

Dentre os principais benefícios do uso de RPA podemos citar os seguintes:

- Disponibilidade

- Os "robôs" trabalham $24 \mathrm{~h}$ por dia e 7 dias por semana de forma ininterrupta.

- Precisão
○ Por serem baseados em regras estruturadas são muito menos suscetíveis a erros.

- Melhora de Moral

- Sem a necessidade de realizar tarefas repetitivas e monótonas os funcionários tem mais tempo a se dedicar a tarefas mais intelectuais.

- Não Invasivo

- Por atuar na camada de apresentação, e ser uma tecnologia não invasiva, a tecnologia não precisa de integração com os sistemas já existentes na corporação.

- Consistência

- Atividades são executadas do mesmo jeito todas vezes, por serem baseadas em regras.

- Auditoria

- Robôs seguem as normas de auditoria a risca e todos possuem histórico auditáveis.

- Produtividade

- Os processos são executados muito mais rapidamente do que quando feito manualmente.

- Barreiras Técnicas

- Não necessárias habilidades de programação para criar ou configurar um robô.

\section{2 TagUI}

TagUI é uma CLI (Command-line tool) para automação de processos (RPA) [15]. Originalmente a ferramenta foi criada por Ken Kosh, especialista em RPA, mas hoje é mantida pela $A I$ Singapore, uma iniciativa governamental de Singapura, criada para estimular a criação habilidades locais de inteligência artificial [15].

O objetivo da ferramenta é reproduzir iterações cognitivas que você possui com websites ou com seu desktop, para que assim o computador possa realiza-las por você, baseada em agendamentos e condições [15].

Ao compararmos a ferramenta com as comercias do mercado, de acordo com a documentação de [15] podemos listar as seguintes vantagens e desvantagens:

Vantagens

- É cross-plataform, ou seja, pode rodar no Windows, macOS ou Linux

- Usuários tem acesso ao código fonte, dessa forma maior segurança

- Disponibilizada sobe licença de uso Open Source Apache 2.0

- Baixa curva de aprendizado, desenvolvimento e deploy rápido

- Fácil adequação a politicas de TI, simplesmente descompactar e usar

- Integração nativa com Python e R para AI/ML/DL

- Integração com API cognitivas Azure/Amazon

\section{Desvantagens}

- Falta de auditoria empresarial, controle, dashboard e relatórios

- Falta de SLA ou time de suporte 24/7

- Time de desenvolvimento pequeno

- Baixa adesão de usuários / desenvolvedores ao longo dos anos

- Falta de consultorias / parceiros para distribuição

\section{Neutro}

- $\quad$ Scripts são escritos em linguagem natural (21 idiomas) e não utiliza fluxogramas 
- Uso de Javascript para desenvolvimento avançado ao invés de C\# / VB
- Automação Desktop baseada em computação visual e OCR

\section{Funcionamento do TagUI}

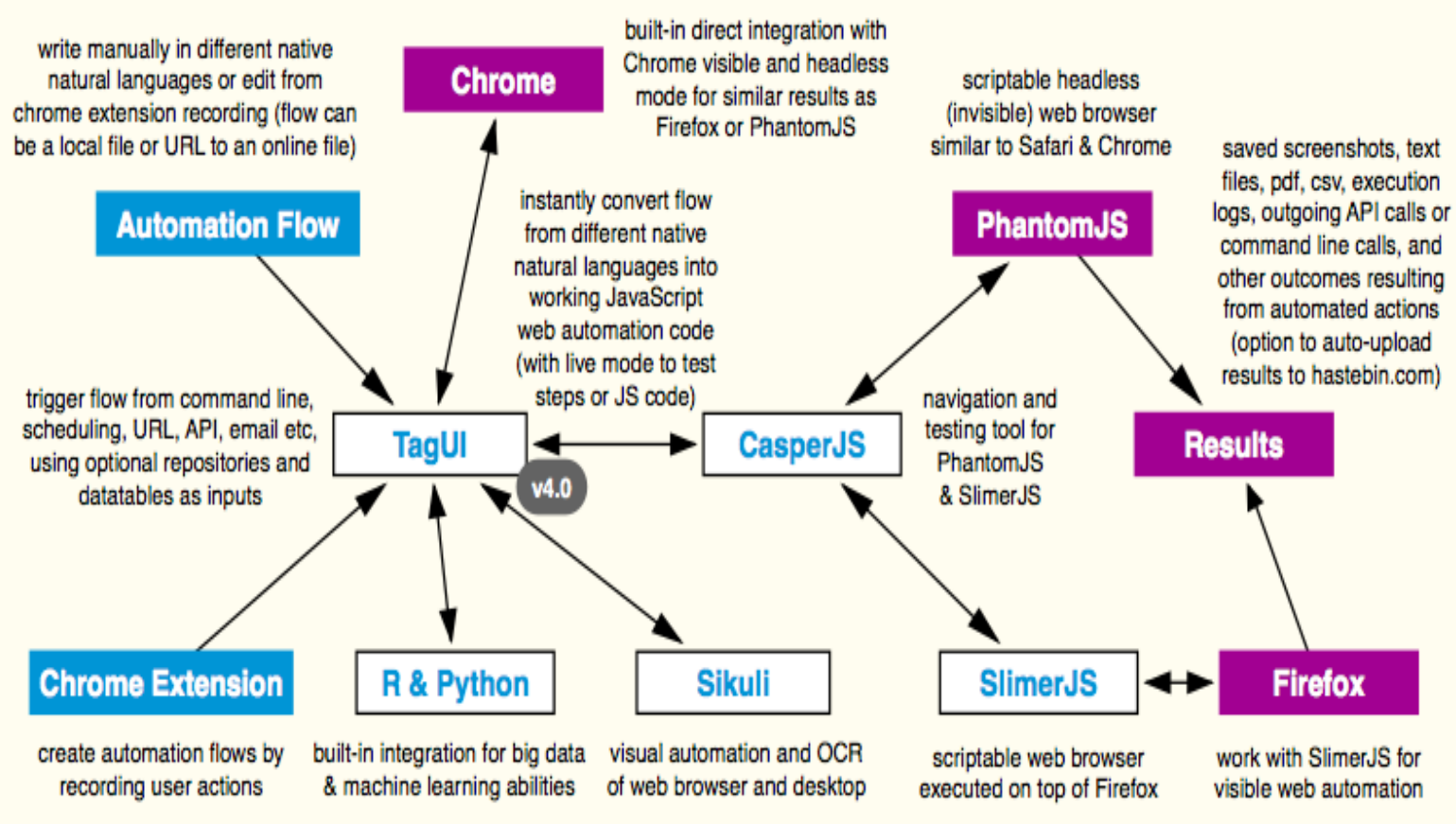

Figura 5 - Arquitetura TagUI.

Fonte: [15].

O processo de automação, que pode ser analisado na Figura 5, acontece da seguinte forma, primeiramente, é necessário criar um script de automação que pode ser criado manualmente ou utilizando a extensão do Chrome.

Após o script criado, existem dois caminhos, se a automação for web ela será convertida da linguagem natural para um Javascript que será utilizado pelo CasperJS, que no processo natural utiliza o PhantomJS para rodar um headless web browser para realizar as ações necessárias. Caso seja passado explicitamente, o CasperJS irá utilizar o SlimerJS que é um browser que permite automação baseado no Firefox.

Em ambos os browsers existe a possibilidade de execução headless que implica que o processo é feito em background ou execução normal, onde é possível ver a automação.

Mais detalhes sobre as funcionalidades podem ser encontrados no fluxo da imagem [15].

\section{- Automation Flow}

É possível desenvolver os scripts manualmente em linguagens naturais, ou gravar os passos com uma extensão do Chrome e edita-lo. O fluxo de automação pode ser local ou passando uma URL para automação online.

\section{- Chrome Extension}

Permite criar automaticamente scripts de automação, com base nas suas ações em uma página web.

\section{- $\quad R \&$ Python}

Integração para execução de scripts para big data e machine learning.
- $\quad$ Sikuli

Automação visual e OCR no browser e no desktop

- CasperJS

Ferramenta para teste e automação baseada em JavaScript para ser utilizada com PhantomJS e SlimerJS.

\section{- PhantomJS}

Headless Browser que permite executar scripts, similar ao Safari e Chrome.

- $\quad$ SlimerJS

Headless Browser que permite executar scripts, compatível com Firefox.

\section{SOLUÇÃO PROPOSTA}

O cenário utilizado como caso de uso de automação é o processo de envio de orçamentos e propostas comerciais de uma empresa de porte médio.

A ideia é ser uma solução de baixo custo e open source. Por esse motivo foi escolhido uma Raspberry Pi para hospedar a aplicação. Mesmo a Raspberry Pi oferecendo uma performance menor que um computador, como será utilizada apenas para essa finalidade, se mostrou o melhor custo benefício.

O processo de automação pode ser descrito com ajuda do fluxo encontrado na Figura 6: 


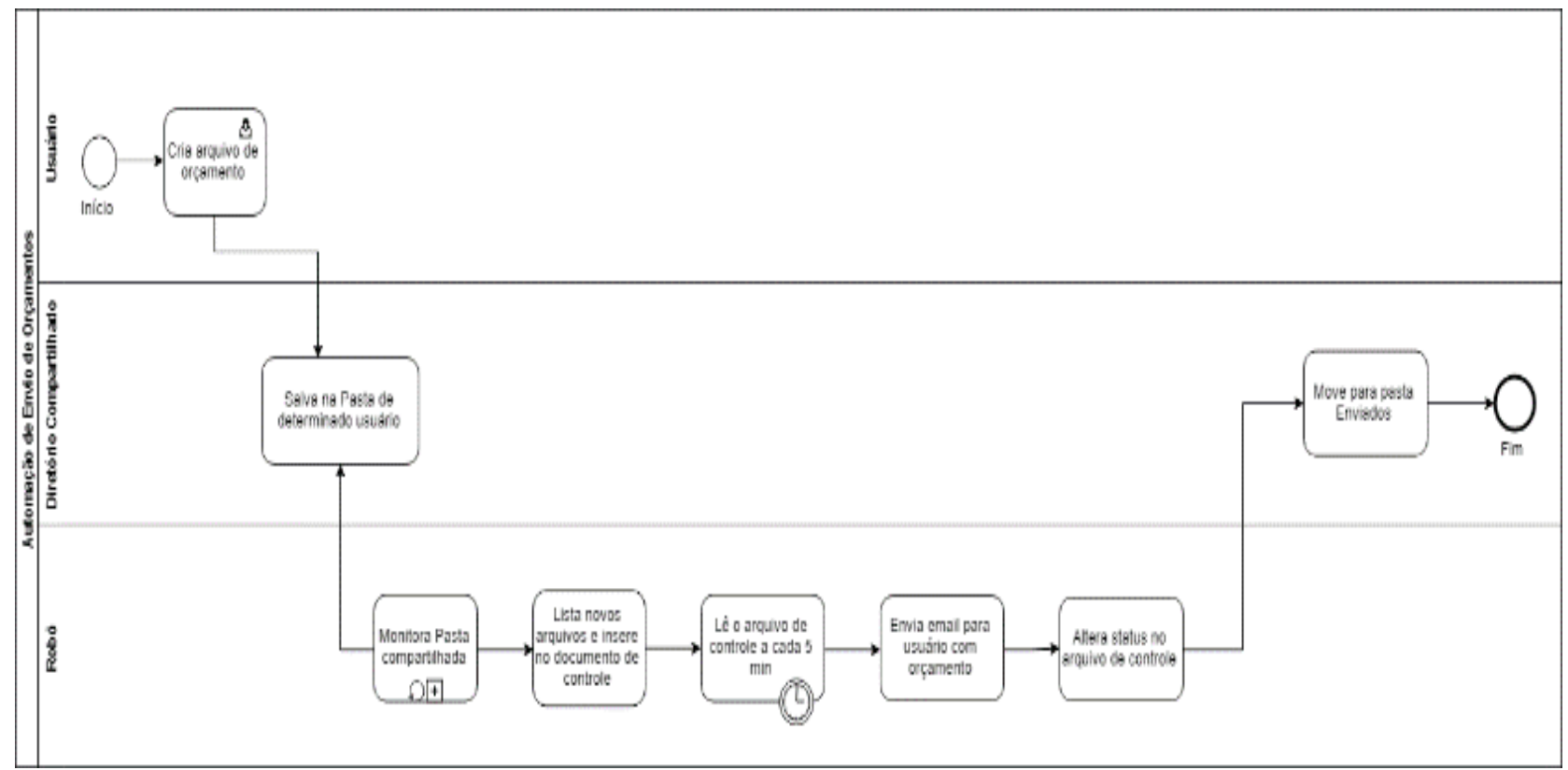

Figura 6 - Fluxograma Robô. Fonte: Autores, (2019).

Onde o usuário cola o arquivo em um diretório, cujo nome é o endereço de e-mail do destinatário. Em paralelo, em uma Raspberry $\mathrm{Pi}$, um script monitora a pasta compartilhada por modificações, no momento em que uma modificação é detectada, se inicia o workflow de automação, seguindo as seguintes etapas:

1. O script extrai o nome e caminho completo dos novos arquivos e insere em um arquivo CSV de controle.

2. A cada 5 minutos, o script criado utilizando o framework RPA TagUI é executado, onde ele itera linha a linha no arquivo CSV checando a coluna "status" do mesmo.

3. Quando o status for igual a "novo" o robô abre o cliente de e-mail e envia ao destinatário.

4. Em seguida altera a coluna "status" do arquivo CSV para "Processado"

5. O último passo é mover o arquivo dentro da pasta compartilhada para uma pasta com nome de "Enviado".

Por ser um framework novo, não existem funções para interagir com arquivos do sistema. Mas ainda assim, por permitir a chamada de scripts Python ou comandos do sistema é possível criar um workflow de automação.

O script descrito no passo 1, foi criado utilizando Python 3.7 e uma biblioteca Watchdog que permite monitorar modificações em um determinado diretório.

Assim como iterações nos arquivos CSV também foram feitas utilizando-se de comandos em Python. Mas o processo de automação da camada de usuário, no caso, do envio de e-mails é utilizando o framework TagUI com sua funcionalidade de visão computacional, com uso da biblioteca Sikulix para automatizar por imagem.

Para o desenvolvimento do script de automação com TagUI é utilizada uma linguagem desenvolvida pelo próprio framework, que deriva uma linguagem natural, como pode ser observado na Figura 7.

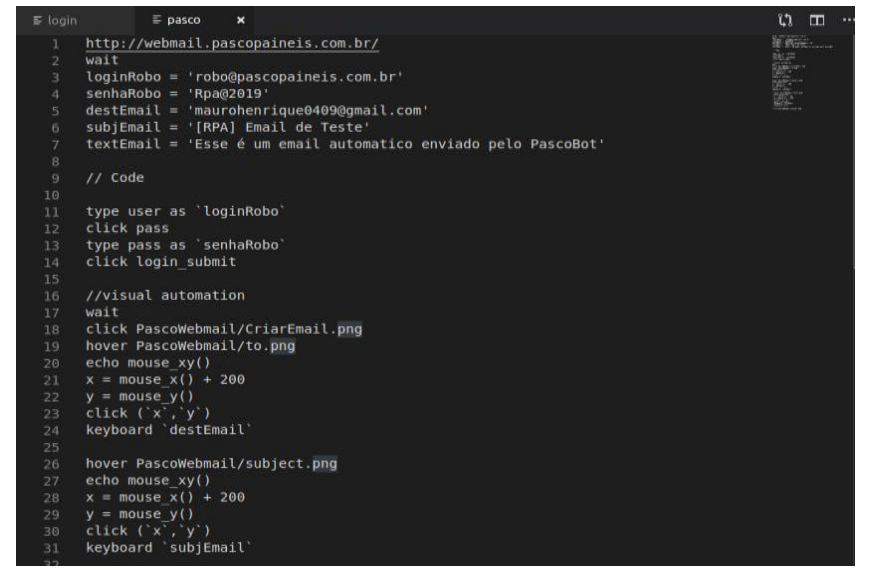

Figura 7 - Código Fonte Automação.

Fonte: Autores, (2019).

Em seguida, esse código é convertido para javascript, como pode ser visto na Figura 8, onde utiliza as bibliotecas CasperJS e Sikulix para realizar as automações necessárias.

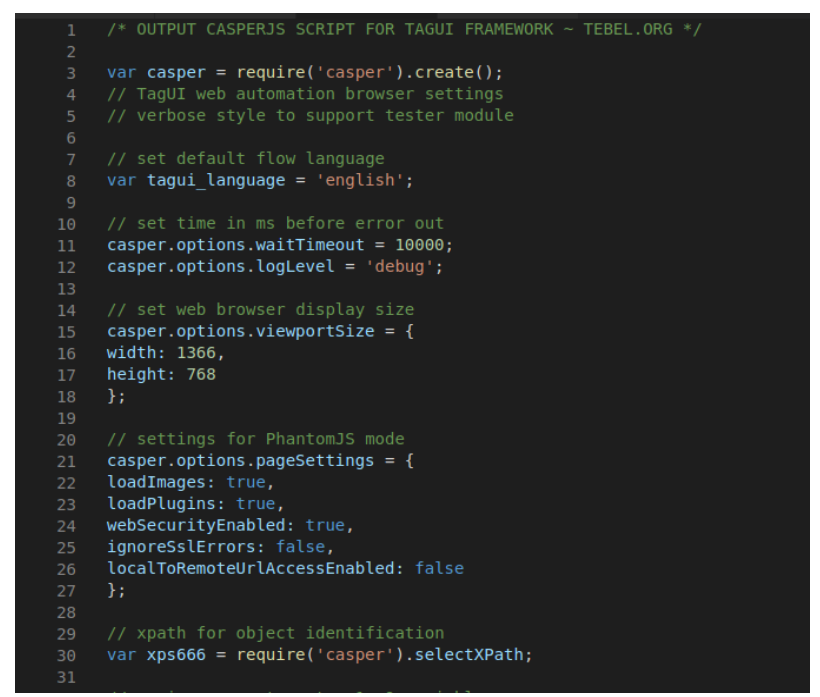

Figura 8 - Código convertido Javacript. Fonte: Autores, (2019). 
Após o fim da execução do robô, fica disponível um arquivo de log (Figura 9), onde é possível verificar cada passo da execução.

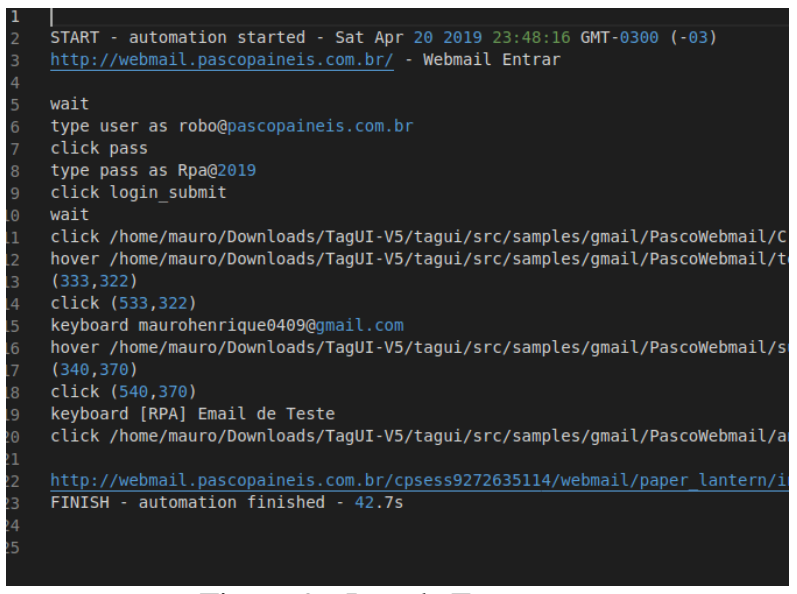

Figura 9 - Log de Execução.

Fonte: Autores, (2019).

\section{RESULTADOS}

Para realizar uma análise de resultado do projeto, foi traçada uma comparação se os benefícios de um projeto RPA foram atingidos.

- Disponibilidade

- O robô pode executar as tarefas $24 \mathrm{~h}$ por dia e 7 dias por semana, bastando apenas ter arquivos a serem processados.

- Precisão

- O processo é executado de acordo com as regras préestabelecidas no código.

- Melhora da Moral

- Os funcionários responsáveis por essa atividade, podem agora focar em atividades mais intelectuais e dinâmicas, como o atendimento aos clientes.

- Não Invasivo

- Toda a automação é feita ou em background ou através da interface gráfica do sistema, dessa forma não precisando de nenhuma integração customizada em aplicações já existentes.

- Consistência

- O robô executa o processo, da forma como foi programada todas as vezes.

- Auditoria

- Nesse ponto, a ferramenta não é ideal, pois gera apenas um arquivo de log. Mas não contém proteção de credenciais e nem uma interface mais dinâmica.

- Produtividade

- Por ser necessário o uso de automação por visão computacional, a execução do processo foi comprometida, em média levando o mesmo tempo que uma pessoa leva para executar, mas algumas vezes travando por não encontrar elementos na tela.

- Barreira Técnica

- Pelo fato, de possuir apenas uma interface de comando, não permitir criação de fluxogramas e nenhum código gráfico. A barreira técnica é alta, necessitando alguém com conhecimentos de desenvolvimento para criar ou realizar a manutenção do robô.

Mesmo não atendendo a todos os benefícios de uma ferramenta de RPA. Por ser um framework em evolução existe grande potencial para que em alguns anos o TagUi se torne amplamente utilizado.

\section{CONCLUSÃO}

O mundo corporativo está passando por uma mudança atualmente, onde as empresas precisam otimizar ao máximo as suas operações. Dentro desse contexto, existem diversas tecnologias que estão desempenhando papeis dentro da chamada "transformação digital", o RPA desempenha um papel muito importante auxiliando as empresas a automatizarem tarefas dentro da sua estrutura. Importante destacar que o RPA possui aplicações diferentes através dos vários segmentos de indústria existentes, mas que geralmente são processos que obedecem algumas características, como por exemplo, alto volume de dados, informações estruturadas e que demandam muito tempo para execução manual. Sendo assim, existem diversos fabricantes no mercado oferecendo ferramentas que permitem a criação de robôs que podem automatizar tarefas repetitivas e tediosas, dessa forma liberando os funcionários para atividades mais desafiadoras e de cunho mais intelectual. Mas é onde encontramos o gap existente, atualmente, a maior parte das ferramentas são proprietárias, de código fechado e dependem de licenciamento de software, que geralmente custam algumas dezenas de milhares de dólares. Dessa forma, o framework TagUi vem como uma alternativa gratuita para desenvolvimento de RPA. Em resultados práticos é possível desenvolver uma automação de tarefas utilizando a ferramenta, onde todos os benefícios tradicionais de uma ferramenta RPA foram alcançados, com exceção do quesito de barreira técnica, pois ainda existe um pré-requisito de conhecimento em linguagens de programação, e a falta de uma interface gráfica que não permite afirmar que ele pode ser comparado a alguma das ferramentas do mercado proprietário. Mesmo assim, por ser um framework novo e em constante evolução, o mesmo está utilizando-se de todas as tecnologias recentes de mercado, como visão computacional e inteligência artificial, sendo possível afirmar que para fins de simples automações é uma ferramenta muito aplicável.

\section{REFERÊNCIAS}

[1] Fersht, Phil. Slaby, James R. Robotic Automation Emerges As A Treat To Tradicional Low-Cost Outsourcing. 2012. Disponível em: $\quad<$ https://www.horsesforsources.com/wpcontent/uploads/2016/06/RS-1210_Robotic-automation-emergesas-a-threat-060516.pdf>. Acessado em: 01/03/2019.

[2] Wilcocks, Leslie. Mary, Lacity. Craig, Andrew. The IT Function and Robotic Process Automation. The Outsourcing Unit Working Research Paper Series [online]. 2015. [data da consulta, 27 de maio de 2019]. Disponível em: < http://eprints.1se.ac.uk/64519/1/OUWRPS_15_05_published.pdf>

[3] Capgemini Consulting. Robotic process automation (RPA): The next revolution of Corporate Functions. 2016. Disponível em: $<$ https://www.uipath.com/solutions/whitepapers/capgemini-nextrevolution>. Acessado em: 30/04/2019.

[4] Brain, David. Fersht, Phil. The RPA Bible: Advanced Topics. $2017 . \quad$ Disponível em: http://info.symphonyhq.com/hubfs/RPABible.pdf $>$. Acessado em: 01/02/2019

[5] Schatsky, David. Muraskin, Craig. Iyengar, Kaunshik. Robotic Process Automation: A path to the cognitive enterprise. Deloite University Press. 2016. Disponível em < https://www.uipath.com/solutions/whitepapers/a-path-to-thecognitive-enterprise >. Consultado em: 01/04/2019. 
[6] C. Moffitt, Kevin. Rozario, Andrea. Vasarhelyi, Miklos. Robotic Process Automation for Auditing. Journal of Emerging Technologies in Accounting [online]. 15. 2018. [data da consulta, 22 de maio de 2019]. Disponível em: <https://aaajournals.org/doi/pdf/10.2308/jeta-10589>

[7] Madakan, Somayya. Holmukhe, Rajesh M., Jaiswal, Durgesh Kumar. The Future Digital Work Force: Robotic process Automation (RPA). Journal of Information Systems and Technology Management [online]. 16. 2019. [data de consulta, 01 de maio de 2019]. Disponível em: < http://www.scielo.br/pdf/jistm/v16/1807-1775-jistm-16e201916001.pdf >

[8] Lacity, Mary C. Willcocks, Leslie P. A new approach to automating services. MIT Sloan Management Review. 2016. [data da consulta, 25 de maio de 2019]. Disponível em: $<$ http://eprints.lse.ac.uk/68135/>

[9] Ostdick. Nick. The Evolution of Robotic Process Automation (RPA): Past, Present and Future. 2016. Disponível em: $<$ https://www.uipath.com/blog/the-evolution-of-rpa-past-presentand-future>. Acessado em: 28/04/2019

[10] Laurent, Patrick. Chollet, Tribault. Herzberg Elsa. Intelligent Automation Entering The Business World. Deloitte Inside Magazine [online]. 2015. 8. [data da consulta, 26 de maio de 2019]. Disponível em:

<https://www2.deloitte.com/content/dam/Deloitte/lu/Documents/ operations/lu-intelligent-automation-business-world.pdf>

[11] van der Aalst, Wil M. P. Bichler, Martin. Heinzl, Armin. Robotic Process Automation. Business \& Information Systems Engineering[online]. 2018. 60. [data da consulta, 27 de maio de 2019]. Disponível em: < https://aisel.aisnet.org/bise/vol60/iss4/1>

[12] IRPA. Introduction To Robotic Process Automation: A Primer. Institute For Robotic Process Automation. 2015. [data da consulta, 22 de maio de 2019]. Disponível em: < https://irpaai.com/wp-content/uploads/2015/05/Robotic-ProcessAutomation-June2015.pdf>.

[13] Lacity, Mary. Willcocks, Leslie. Robotic Process Automation: The Next Transformation Lever for Shared Services. The Outsourcing Unit Working Research Paper Series [online]. 2016. [data da consulta 21 de maio de 2019]. Disponível em: <http://www.umsl.edu/ lacitym/OUWP1601.pdf>

[14] Schmidt, Daniel. 10 RPA Use Cases for IT, HR, Finance \& Accounting and more. 2018. Disponível em: < https://www.kofax.com/Blog/2018/november/10-rpa-use-casesfor-it-hr-finance-accounting-and-more . Acessado em: 04/05/2019.

[15] TagUi. Readme. 2019. Disponível em: < https://github.com/kelaberetiv/TagUI/blob/master/README.md

> Acessado em: 01/03/2019. 